\title{
APPLICATION OF COMPLEX RESISTIVITY TOMOGRAPHY TO FIELD DATA FROM A KEROSENE-CONTAMINATED SITE
}

\author{
Andreas Kemna, Eiko Räkers \\ DMT-GeoTec, Geo-Engineering, Bochum, Germany
}

Andrew Binley

CRES, Institute of Environmental and Biological Sciences, Lancaster University, England

\section{INTRODUCTION}

Recently several investigations on samples in the laboratory have proved the influence of hydrocarbon $(\mathrm{HC})$ contamination on the complex electrical resistivity of soils and rocks (Olhoeft, 1992; Börner et al., 1993; Vanhala, 1997). Beside the magnitude of resistivity additional information about the contaminant is obtained from the phase angle which may be measured by the frequency domain induced polarization method. However, for environmental HC pollution problems the importance of the complex resistivity method depends on its practical value for the detection, delineation and monitoring of subsurface contamination under field conditions. Here, an application of complex resistivity tomography to field data, collected on a kerosene-contaminated former military jet fuel depot, is presented.

\section{SITE DESCRIPTION}

The site is located next to the Strasbourg-Entzheim airport, France. The general geological stratification is well known from cored drillings. Below a top loessic layer about $1.5 \mathrm{~m}$ thick follow sands and gravels in which, at a depth of approximately $9 \mathrm{~m}$, a nearly $1 \mathrm{~m}$ thick clay layer is embedded just above the water table (see Figure 1). The military fuel depot was built in 1957, since then the soil was strongly contaminated by kerosene, probably due to slow leakage of the underground storage tanks over a long time. With regard to the characteristic penetration of light non-aqueous phase liquids it was expected that the contaminant moved downward through the unsaturated zone to the top of the low-permeable clay layer, or even the water table, following the most permeable pathways. From there, a lateral spreading in the direction of ground water flow and the development of dissolved contaminant plumes may have occured. Application of complex resistivity tomography was hoped to give more insight into the contaminant migration pattern.

For the electrical measurements four boreholes, each equipped with 16 equally spaced copper electrodes, were drilled. In addition, 30 electrodes were placed at the surface defining three vertical planes for the tomographic reconstruction. The field setup is shown in Figure 1. Altogether about 3000 dipole-dipole resistivity and chargeability data were collected in October, 1996. Since the measurements were carried out in the time domain, the apparent chargeability values were converted to appropriate phase angles of a complex apparent resistivity using the procedure described below. 

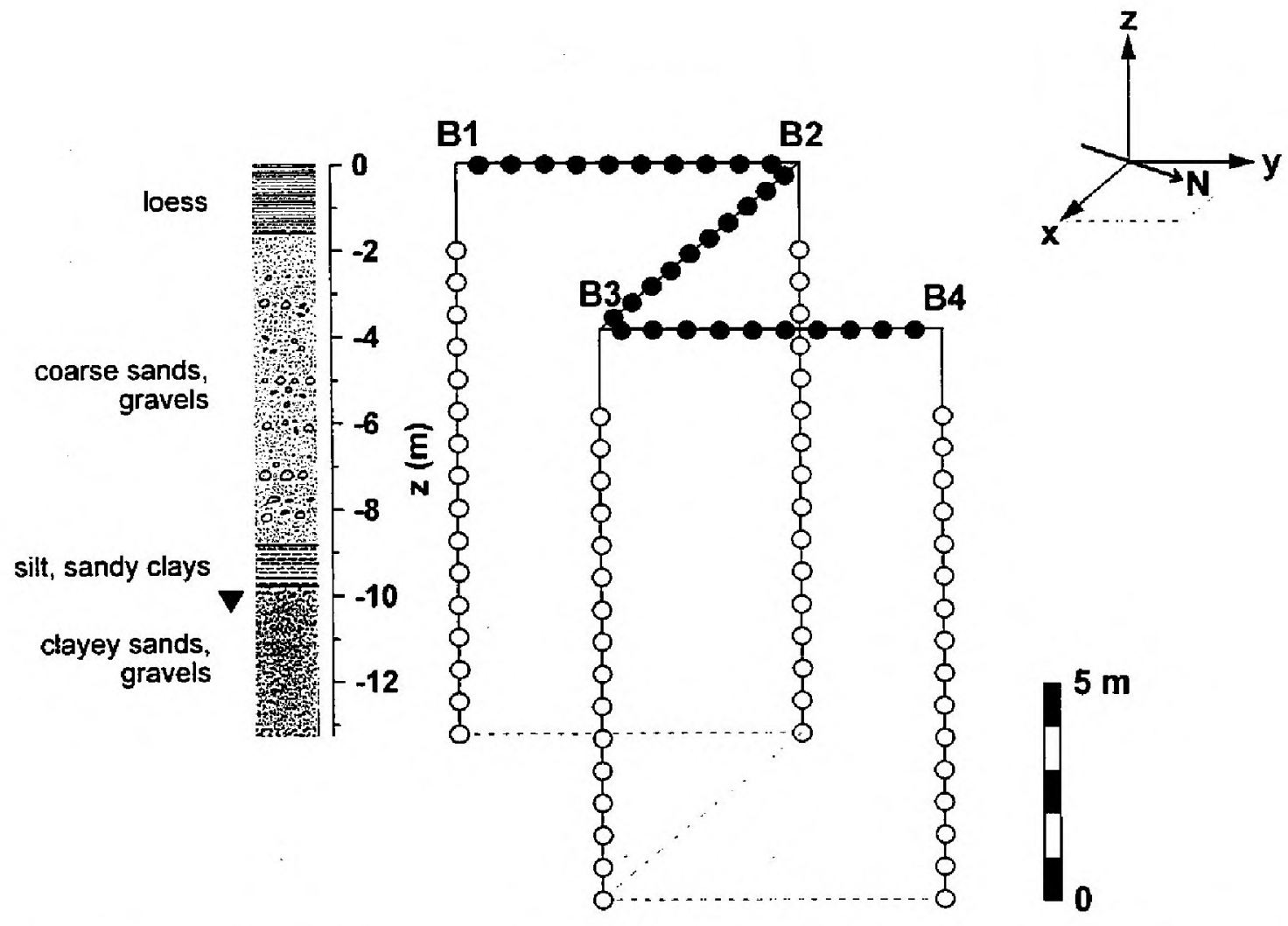

Figure 1: Three-dimensional view of the four boreholes B1, B2, B3 and B4. Solid and open circles indicate position of surface and borehole electrodes, respectively. In addition, the geological stratification known from the fully recovered cores at borehole Bl is shown.

\section{CONVERSION OF CHARGEABILITY TO PHASE ANGLE}

For many situations the function

$$
\rho(\omega)=\rho_{0}\left(\frac{\omega_{0}}{i \omega}\right)^{h}=|\rho(\omega)| \mathrm{e}^{i \varphi}
$$

describes well the observed spectral behaviour of intrinsic complex resistivity (Van Voorhis et al., 1973; Börner et al., 1993). In (1) $\mathrm{i}$ is the imaginary unit, $\omega$ is the angular frequency, and $\rho_{0}$ is the magnitude of the resistivity at a reference frequency $\omega_{0}$. The frequency exponent $b$ is directly related to the constant phase angle $\varphi=-\pi b / 2$.

For low contrasts within the intrinsic phase angles the same model may be applied to the apparent resistivity $\rho_{\mathrm{a}}(\omega)$. Then, integrating the inverse Laplace transform of (1) with regard to time yields the time domain step response, from which the response due to a square current waveform can be synthesized by superposition. For the measured voltage at time $t$ after switch-off of $N$ alternating positive and negative pulses of magnitude $I_{0}$ and duration $T$, with successive pulses being separated by an off period of $T$, one obtains the series

$$
U(t)=\frac{I_{0} \rho_{\mathrm{a} 0} \omega_{0}^{b}}{K b \Gamma(b)} \sum_{n=0}^{N-1}(-1)^{N-n}\left[(t+2 n T)^{h}-(t+T+2 n T)^{b}\right] .
$$

In (2) $\Gamma$ and $K$ denote the Gamma function and the geometric factor of the electrode arrangement, respectively. Obviously, the measured apparent chargeability can be related to the frequency exponent $b$, and hence to an apparent phase angle $\varphi_{\mathbf{a}}$, using equation (2) with $\omega_{0}=\pi / 2 T$. 


\section{INVERSION ALGORITHM}

From the datasets belonging to the different planes the underlying distribution of intrinsic complex resistivity was reconstructed by application of a regularized two-dimensional inversion algorithm based on full complex arithmetic that is basically described in Kemna and Binley (1996). Because of the known geology, in addition an anisotropic smoothing was adopted from Oldenburg and $\mathrm{Li} \mathrm{(1994)} \mathrm{that} \mathrm{causes} \mathrm{a} \mathrm{light} \mathrm{estimation} \mathrm{of} \mathrm{horizontal} \mathrm{structures}$ within the inversion process.

The individual data errors were estimated by comparison of normal and reciprocal measurements. To account for outliers in the data the robust estimation method proposed by Morelli and LaBrecque (1996) was implemented. The procedure is based on a certain increase of the errors belonging to those data which are poorly fit by the reconstructed model at each inversion iteration. Here it is applied to the magnitudes of the complex data errors since these determine the weighting terms within the complex inversion.

\section{INVERSION RESULTS AND INTERPRETATION}

The results of the inversion for the different planes are shown in Figure 2. The inverted images of resistivity magnitude clearly show the conductive clay layer. Both depth and thickness correspond well with the recovered drill cores (see Figure 1). Within the sand/gravel region the magnitude increases slightly towards the boreholes suggesting a geometric artefact. The reconstructed phase distributions show a background phase lag for the sand/gravel fraction of about $10 \mathrm{mrad}$. The top loessic layer can be identified throughout the images as a low-polarizable region.

Between the boreholes $\mathrm{B} 3$ and $\mathrm{B} 4 \mathrm{a}$ further significant anomaly of decreased phase shift extends from approximately $y=13.5 \mathrm{~m}, z=-7.5 \mathrm{~m}$ to $\mathrm{y}=9.5 \mathrm{~m}, \mathrm{z}=-10.5 \mathrm{~m}$. It crosses the clay layer, which is located at a depth of 8.3 to $9.7 \mathrm{~m}$. According to Vanhala (1997), one possible explanation for the decrease of phase shift could be that the contaminant detached ions from the particle surfaces to the electrolyte at residual saturation within the pore spaces. This causes a decrease of the thickness of the electrical double layer at the interface between the solid matrix and the electrolyte resulting in a reduced polarization, and hence in a decreased phase lag in these regions. Consequently, the decreased phase shift would indicate kerosenecontaminated areas or, at least, areas with a relatively high contaminant concentration. This interpretation would be in accordance with the odour of hydrocarbons observed within borehole B4 from a depth of $7 \mathrm{~m}$. Moreover, the jet fuel would already have penetrated through the low-permeable clay layer being in contact with the ground water. The slight extent of the -anomaly below the water table could be caused by seasonable water table fluctuations or dissolved contaminant components. The complete migration pathway would coincide with the direction of ground water flow supporting the given explanation.

The presented results of the complex resistivity tomography regarding contaminant detection will be compared with a detailed chemical analysis of the recovered core samples from the drillings.

\section{ACKNOWLEDGEMENTS}

This work is part of the research project "Detecting and locating non-aqueous phase contaminants in soils by three geophysical methods" supported by the European Environment and Climate Programme (Contract ENV4-CT95-0079). 


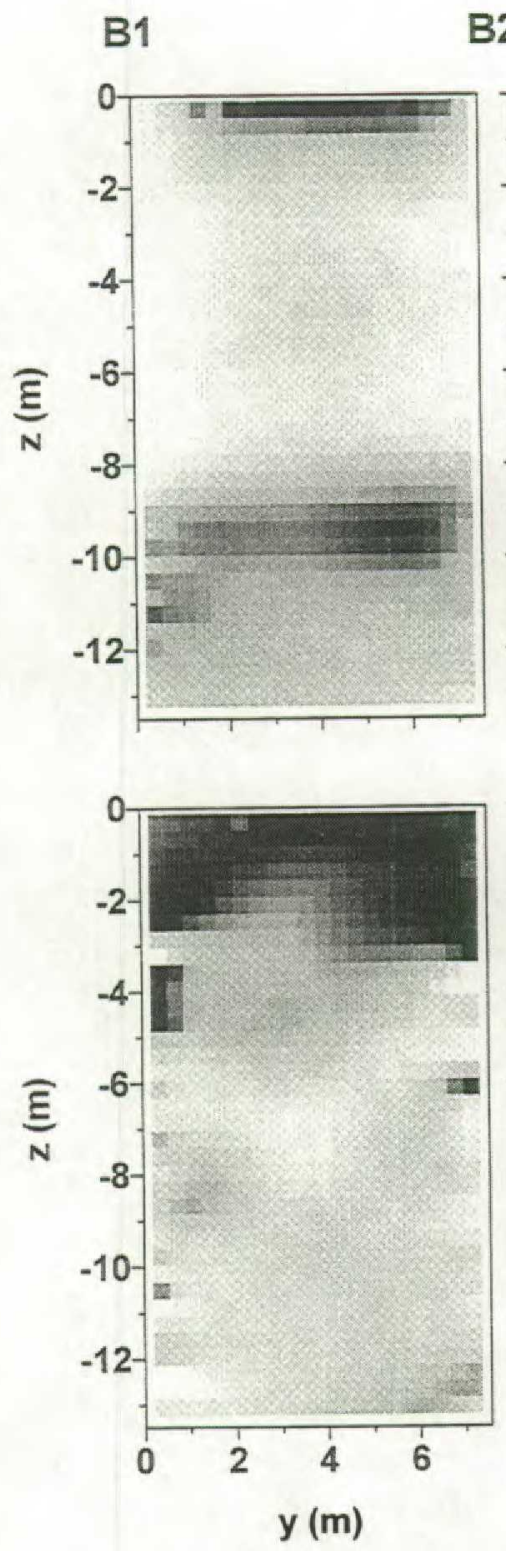

B2 B3

B4.
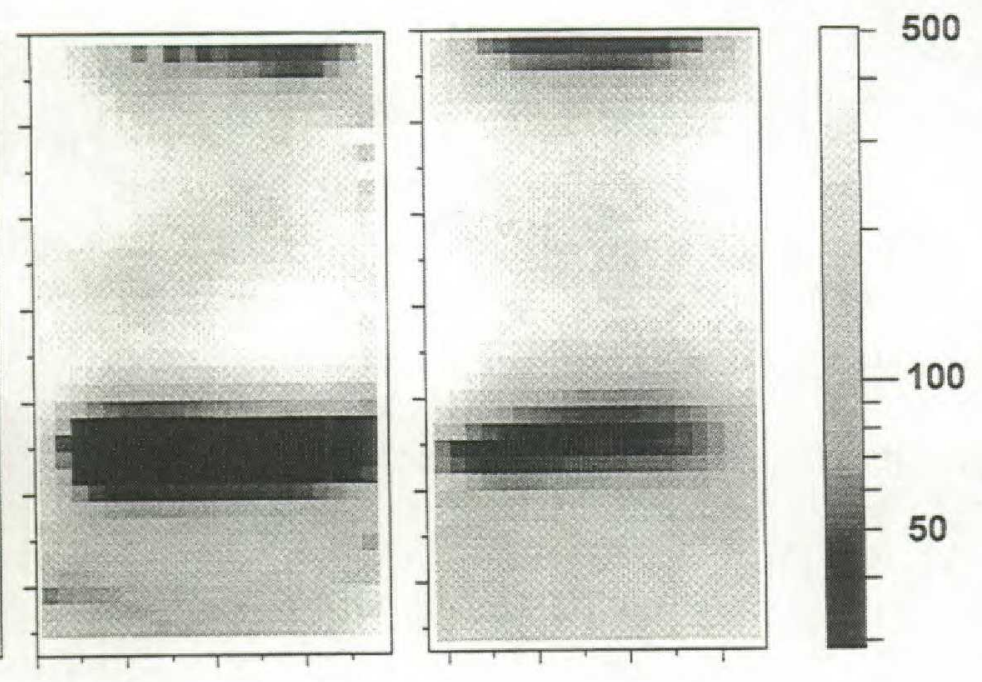

$|\rho|(\Omega \mathrm{m})$

Figure 2: Results of complex resistivity inversion showing magnitude and phase in the different reconstruction planes.

\section{REFERENCES}

Börner, F., Gruhne, M., and Schön, J., 1993, Contamination indications derived from electrical properties in the low frequency range: Geophys. Prosp., 41, 83-98.

Kemna, A., and Binley, A., 1996, Complex electrical resistivity tomography for contaminant plume delineation, in Lagabrielle, R., Ed., Proc. 2nd Mtg. Environmental and Engineering Geophysics: EEGS, European section, Nantes, 2nd-5 September 1996, 196-199.

Morelli, G., and LaBrecque, D. J., 1996, Advances in ERT inverse modeling: European J. of Environmental and Engineering Geophysics, 1, 171-186.

Oldenburg, D. W., and Li, Y., 1994, Inversion of induced polarization data: Geophysics, 59, 1327-1341.

Olhoeft, G. R., 1992, Geophysical detection of hydrocarbon and organic chemical contamination, in Bell, R. S., Ed., Proc. Symposium on the Application of Geophysics to Engineering and Environmental Problems: EEGS, 26-29 April 1992, 587-596.

Vanhala, H., 1997, Mapping oil-contaminated sand and till with the spectral induced polarization (SIP) method: Geophys. Prosp., 45, 303-326.

Van Voorhis, G. D., Nelson, P. H., and Drake, T. L., 1973, Complex resistivity spectra of porphyry copper mineralization: Geophysics, 38, 49-60. 